\title{
ON THE STORAGE-DISCHARGE RELATIONSHIPS IN THE FLAT LAND
}

\author{
By Md. Sayeedul Islam KHAN*, Fusetsu TAKAGI** \\ and Shohei ADACHI***
}

\section{INTRODUCTION}

On the runoff process in the flat land a number of research works are found. In most of these works the lumped models have been treated for the practical uses. As for example, considering the overland and channel regions as a reservoir or a $\operatorname{tank} \mathrm{k}^{7}$, the so-called storage function, i.e. the storage-discharge relationship has been widely accepted. The characteristics of the storage function have been examined empirically in many of the actual watersheds. These have also been discussed theoretically with the help of the uniform flow hydraulics. However, on the flat land the flow depends exceedingly on the boundary conditions in the actual watersheds and the uniform flow does not take place in a flat land with horizontal bed. Therefore, no such definite relationships have yet been found with respect to the runoff phenomena in the watershed with no bed slope.

As another approach, the kinematic wave method $^{6), 8), 9)}$ has been also applied to discuss the flow behaviours in the flat land. As this method is based on the quasi-uniform assumption, this assumption can not be applied for the flow over the basin with horizontal bed.

In this situation, the authors have attempted to clarify the runoff characteristics in the flat land in connection with the hydraulic behaviours of water flow within a watershed. A part of the research has already been published ${ }^{3}$. In the previous paper $^{3}$, the free fall condition at the

* Members of JSCE Assoc. Prof., Dep. of Civil Engineering, Rajshahi Engineering College, Bangladesh.

** Member of JSCE Prof. Dr., Dept. of Civil Engineering, Nagoya Univ.

*** Member of JSCE Prof. Dr., Dept. of Civil Engineering, Nagoya Univ. outlet of watershed was chosen as the subject of the study and then the storage-discharge relationship in the flat land had been introduced in a simple form by solving the hydraulic equations as to the overland flow and channel systems. Moreover, it was pointed out that the relationships may be evaluated in terms of non-dimensional parameters $\epsilon$ and $I$.

In the present paper, the authors will discuss if such identical storage-discharge relationships exist on the runoff in the flat land with some other boundary conditions, and if so, how the previous relationship should be modified and evaluated for these new boundary conditions. Realizing the drainage systems in the actual flat land, discussions have been carried out on the two types of boundary conditions, i.e. i) the drainage system with a weir at its outlet, and ii) the drainage is controlled by the constant water level at the outlet. What we would especially like to emphasize now is the fact that the runoff characteristics, which could not be answered by the conventional approach of the lumped system model or a kinematic wave method, have been systematically clarified in terms of the non-dimensional parameters in the light of the hydraulic behaviours within the watershed.

\section{FUNDAMENTAL EQUATIONS}

In the case of the overland flow the lateral inflow is the rainfall, but for the channel flow the outflow from the overland is considered as the only lateral inflow. The fundamental flow equations ${ }^{1,3), 5)}$ are derived from the differential form of the continuity and momentum equations. These flow equations when applied to the overland and the channel runoff problems and transformed to the non-dimensional quantities ${ }^{3)}$ take the following forms for the unit width of flow.

$$
\frac{\partial H_{j}}{\partial T_{j}}+\frac{\partial\left(U_{j} H_{j}\right)}{\partial X_{j}}=\theta \cdot R_{j},
$$




$$
\frac{\partial U_{j}}{\partial T_{j}}+U_{j} \frac{\partial U_{j}}{\partial X_{j}}+\epsilon_{j}\left(\frac{\partial H_{j}}{\partial X_{j}}-I_{j}\right)+\lambda \frac{U_{j}^{2}}{H_{j}^{4 / 3}}=0,
$$

where $X$ : non-dimensional distance along the flow plane, $T$ : time, $H$ : depth of flow, $U$ : velocity of flow, $R$ : lateral inflow, $I$ : slope and $\theta, \epsilon$ and $\lambda$ are dimensionless coefficients. The suffices $j=1,2$ indicate the quantities for the overland and the channel flows respectively.

The relations between the non-dimensional and dimensional quantities through their normalizing operators ${ }^{3), 4)}$ are shown below.

$$
\begin{aligned}
& X=x / x^{*}, \quad T=t / t^{*}, \quad H=h / h^{*}, \\
& U=\frac{u}{x^{*} / t^{*}}, \quad I=\frac{i}{h^{*} / x^{*}}, \quad R_{j}=r j / r^{*},
\end{aligned}
$$

where $x, t, h, u, i$ and $r$ are the corresponding dimensional quantities and $x^{*}, t^{*}, h^{*}$ and $r^{*}$ are the normalizing operators for the quantities of distance, time, depth and lateral inflow, respectively. The dimensionless coefficients $\theta, \epsilon$ and $\lambda$ may be expressed ${ }^{3)}$ in the following relationships with the normalizing operators.

$$
\theta=r^{*} \cdot \frac{t^{*}}{h^{*}}, \quad \epsilon=g h^{*} \cdot \frac{t^{* 2}}{x^{* 2}}, \quad \lambda=g n^{2} \frac{x^{*}}{h^{* / 3}},
$$

where $g$ : acceleration due to gravity, $n$ : Manning's roughness coefficient.

The normalizing operator $x^{*}$ is concerned here with the field or channel length $L$. As such, by considering $x^{*}=L$ and setting both $\theta$ and $\lambda$ equal to unity, the normalizing operators $h^{*}$ and $t^{*}$ are expressed as

$$
h^{*}=\left(g n^{2} L\right)^{3 / 4}, \quad t^{*}=\frac{\left(g n^{2} L\right)^{3 / 4}}{r^{*}} .
$$

Then, the parameters $\epsilon$ and $I$ may be evaluated as

$$
\epsilon=\frac{g^{13 / 4} n^{9 / 2} L^{1 / 4}}{r^{* 2}}, \quad I=\frac{i L^{1 / 4}}{g^{3 / 4} n^{3 / 2}} .
$$

And Eqs. (1) and (2) may be rewritten as

$$
\begin{aligned}
& \frac{\partial H_{j}}{\partial T_{j}}+\frac{\partial\left(U_{j} H_{j}\right)}{\partial X_{j}}=R_{j}, \\
& \frac{\partial U_{j}}{\partial T_{j}}+U_{j} \frac{\partial U_{j}}{\partial X_{j}}+\epsilon_{j}\left(\frac{\partial H_{j}}{\partial X_{j}}-I_{j}\right)+\frac{U_{j}^{2}}{H_{j}^{4 / 3}}=0 .
\end{aligned}
$$

In the expression for lateral inflow in Eq. (3), $R_{1}$ for the overland corresponds to the nondimensional rainfall excess and $R_{2}$ for the channel corresponds to the non-dimensional overland outflow $Q_{1}$. Again, as the widths of flow in the overland and in the channel systems are different, the magnitudes of $r^{*}$ are also different for the two systems.

$$
r_{1}^{*}=\bar{r}, \quad \text { for the overland }
$$

and

$$
r *_{2}=\tilde{r} \beta \text {, for the channel, }
$$

where $\bar{r}$ : mean rainfall excess, and $\beta$ : ratio of the overland flow length $L_{1}$ to the channel width $b_{2}$.

\section{INITIAL AND BOUNDARY CONDITIONS}

The following three cases are considered and investigated in this study. Practicability of these boundary conditions is discussed later in the chapter 6 .

\section{(1) Case I: Weir at the outlet:}

Flow behaviours due to a) the weir at the overland outlet and b) free fall at the overland outfall and the weir at the channel outlet are considered. Figures 1(a) and 1(b) show the elemental watershed models with weir at the overland and the channel outlets, respectively. The weirs have been considered as broad-crested and as such

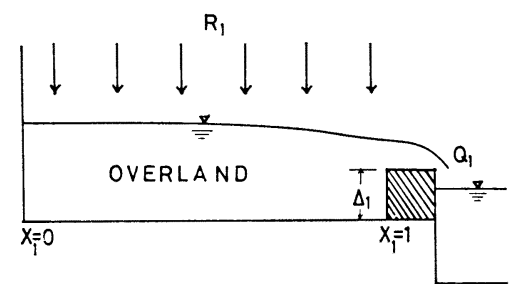

Fig. 1 (a) Model picture of overland runoff with weir at the outlet.

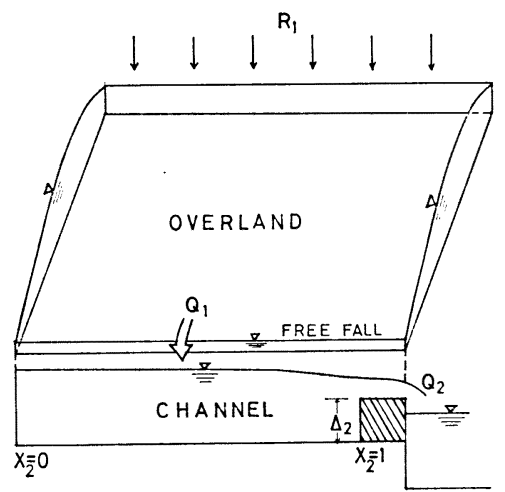

Fig. 1 (b) Model picture of runoff with free fall at the overland outlet and weir at the channel outlet. 
critical condition of flow occurs over the weirs. With weir at the outlet, the boundary conditions may be given by

$$
\begin{array}{ll}
U_{j}=0 & \text { at } X_{j}=0, \\
U_{j}=\sqrt{\epsilon_{j}\left(H_{j}-\Delta_{j}\right)} & \text { at } X_{j}=1,
\end{array}
$$

where $\Delta_{j}$ : dimensionless weir height. If the dimensional weir height is denoted by $\delta$, then

$$
\Delta_{j}=\delta_{j} / h^{*}{ }_{j} .
$$

For the flow behaviour over weirs some correction factor should actually be applied for the velocity at the downstream end. And if we need to take care of such a factor, we may consider the effect in the evaluation of the value of $\epsilon j$.

Two types of initial condition-one dry and the other with water depth upto the weir crestare considered, i.e.

$$
\begin{array}{lll}
U_{1}=0, & H_{1}=0 & \text { at } T_{1}=0, \\
U_{j}=0, & H_{j}=\Delta_{j} & \text { at } T_{j}=0 .
\end{array}
$$

\section{(2) Case II: Constant water depth at the out- let}

Flow behaviour due to a) the constant depth of water at the overland outlet, and b) free fall at the overland outfall and the constant depth of water at the channel outlet is considered.

Figures 2(a) and 2(b) show the elemental model of runoff process with the constant depths of water at the overland and the channel outlets, respectively. The upstream and the downstream boundary conditions may be given by

$$
\begin{array}{ll}
U_{j}=0 & \text { at } X_{j}=0, \\
H_{j}=H_{o j} & \text { at } X_{j}=1.0,
\end{array}
$$

where $H_{0}$ : the constant water depth. The initial condition considered is

$$
H_{j}=H_{o j}, \quad U_{j}=0 \quad \text { at } T_{j}=0 .
$$

\section{(3) Case III: Flow behaviour at intermediate sections with the free fall at the outlet}

The model of flow behaviour at the intermediate sections with free fall at the outlets of both overland and channel is shown in Fig. 3. The following upstream and downstream boundary conditions are assumed.

$$
\begin{array}{ll}
U_{j}=0 & \text { at } X_{j}=0, \\
U_{j}=\sqrt{\epsilon_{j} H_{j}} & \text { at } X_{j}=1 .
\end{array}
$$

The initial condition considered is

$$
U_{j}=0, \quad H_{j}=0 \quad \text { at } T_{j}=0 .
$$

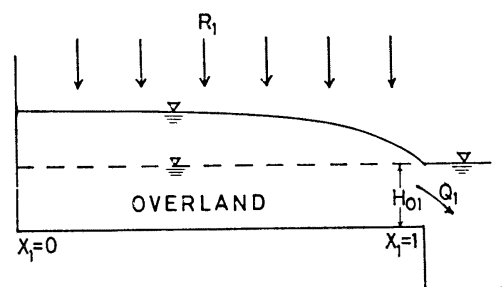

Fig. 2 (a) Model picture of overland runoff with constant water depth at the outlet.

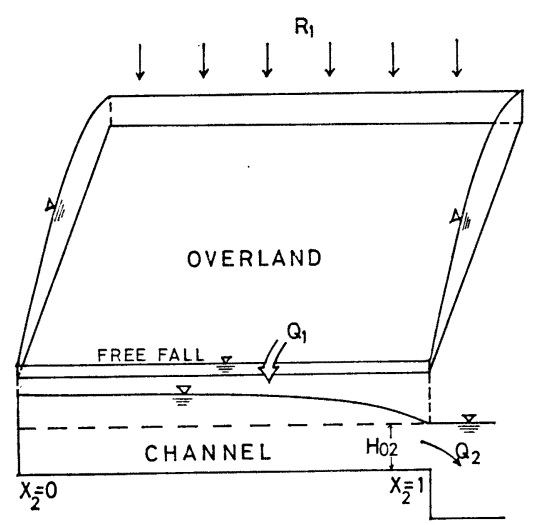

Fig. 2 (b) Model picture of channel runoff with free fall at the overland outlet and constant water depth at the channel outlet.

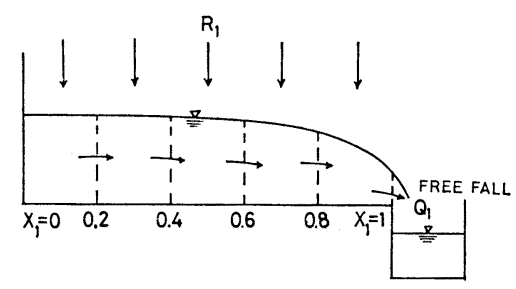

Fig. 3 Model picture of runoff at the intermediate sections of overland with free fall at the outlet.

\section{NUMERICAL SOLUTION AND FLOW PARAMETERS}

The solution of the overland and the channel flow equations is obtained with the help of 2step Lax-Wendroff method $^{2}$ in the same manner as in the previous paper.

The stability of the solution is checked by the condition given by Von-Neumann ${ }^{2}$. The equation suggested by Von-Neumann takes the follow- 
ing form when converted to non-dimensional terms.

$$
\frac{\partial T_{j}}{\partial X_{j}}\left(\left|U_{j}\right|+\sqrt{\epsilon_{j} H_{j}}\right) \leq 1 .
$$

The stability of the solution for all the three cases may be checked by the above equation after noting that i) for the Case I, at the outlet, $H_{j}=$ $\left(H_{j}-\Delta_{j}\right)$ and ii) for the Case II, at the outlet, $H_{j}=H_{o j}$.

There are two non-dimensional parameters $\epsilon_{j}$ and $I_{j}$ in Eqs. (7) and (8). These parameters are defined by Eq. (6). The magnitude of $\epsilon_{1}$ for an usual overland basin is expected to be within the range $10^{6} \sim 10^{8}$ as discussed in the previous paper. For the channel, the corresponding magnitude of $\epsilon_{2}$ lies between $10^{2} \sim 10^{4}$. For the slope parameter $I_{j}$ it is considered here that $I_{1}=I_{2}=0$ (horizontal bed). Rainfall of constant intensity has been considered in this study and as such $R_{1}=1.0$ during the rising stage and $R_{1}=$ 0.0 during the recession period.

With weir at the downstream end (Case I) there is a new parameter $\Delta_{j}$ in Eq. (11). The nondimensional weir height $\Delta_{j}$ is defined by Eq. (12). The magnitude of $\Delta_{1}$ at the overland outlet is varied between the range $0<\Delta_{1} \leq 0.06$ and $\Delta_{2}$ at the channel outlet is considered between the range $0<\Delta_{2} \leq 0.32$. The upper limit considered here corresponds to the case when the equilibrium water surface becomes practically horizontal and the equilibrium live storage becomes equal to the equilibrium critical depth. Live storage is defined by the expression $S_{L_{j}}=\int_{0}^{1}\left(H_{j}-\Delta_{j}\right) d X$.

For the Case II, the new parameter in the downstream boundary condition is the nondimensional constant depth of water $H_{o j}$ as shown in Eq. (14). The magnitudes of $H_{o j}$ are varied within the range as shown below.

$$
0.002 \leq H_{o 1} \leq 0.06 \text {, and } 0.05 \leq H_{o 2} \leq 0.6 \text {. }
$$

The lower limit of $H_{o j}$ indicates the equilibrium critical depth under free fall condition and the upper limit corresponds to the case when the water surface is almost horizontal and the equilibrium storage $S_{\mathrm{max}} \simeq H_{o j}$.

The non-dimensional storage may be expressed in terms of the dimensional storage and the normalizing operators in the following manner.

$$
S_{j}=\int h_{j} d x_{j} / h_{j} * x_{j} *
$$

\section{FLOW BEHAVIOUR AND STORAGE- DISCHARGE RELATIONSHIP}

\section{(1) Case I: Weir at the outlet}

Figure 4 shows the water profile for an overland basin of $\epsilon_{1}=10^{7}$ corresponding to several magnitudes of $\Delta_{1}$. The water profiles are considered in Fig. 4 when flow condition reaches equilibrium. It is seen from this figure that with increasing $\Delta_{1}$ the water surface tends to become horizontal.

With weir at the overland outlet two types of initial conditions are considered here before the commencement of rainfall. In one case the basin is considered dry and for the other case the basin is full with water upto the crest of the weir. Figure 5 shows the discharge hydrographs for the dry overland basin of $\epsilon_{1}=10^{7}$ with respect to several magnitudes of $\Delta_{1}$. Some time lag is noticed at the initial stage of the discharge hydrographs. This is because of the fact that the basin is dry and some initial period is required to fill up the basin upto the crest of the weir before

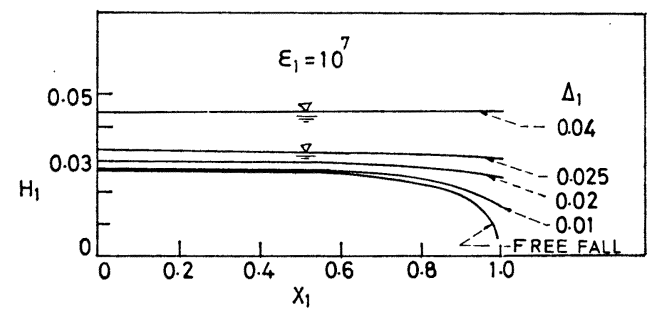

Fig. 4 Equilibrium water profile of overland flow with weir at the outlet.

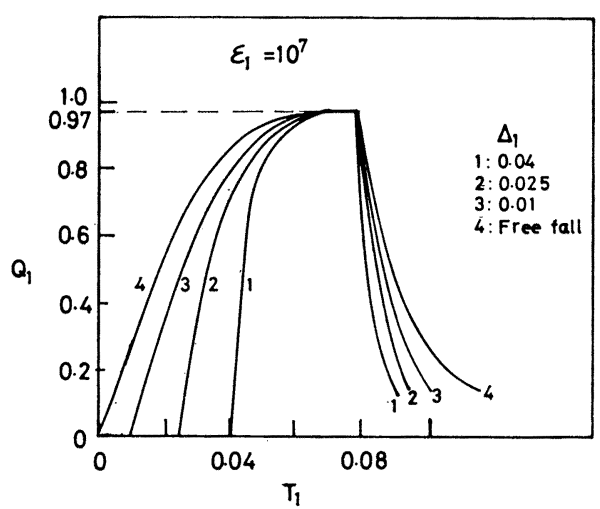

Fig. 5 Overland discharge hydrograph with weir at the outlet for an initially dry basin. 


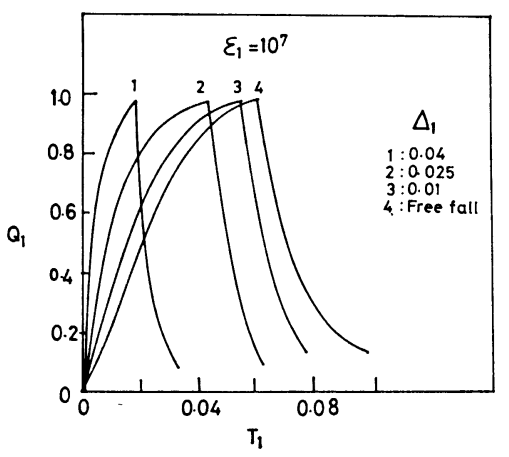

Fig. 6 Overland discharge hydrograph with weir at the outlet with the initial condition $H_{1}=\Delta_{1}$.

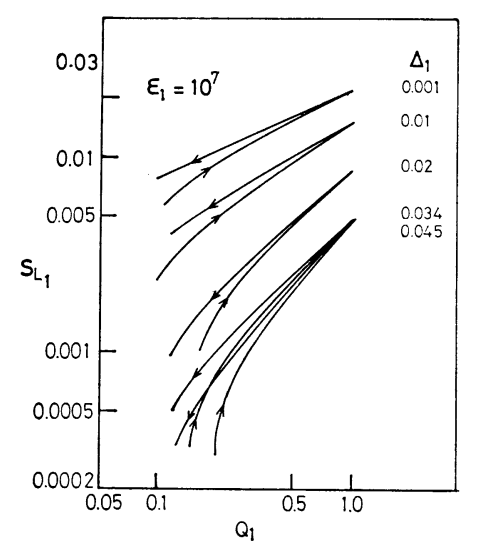

Fig. 7 Logarithmic storage-discharge relation with weir at the overland outlet.

the actual flow starts. Figure 6 shows the discharge hydrograph for the same basin but with initial water depth upto the crest of the weir. It is seen from this figure that the discharge hydrograph tends to become steeper and the time to peak decreases with increasing $\Delta_{1}$.

Figure 7 shows the overland storage-discharge relation for $\epsilon_{1}=10^{7}$ corresponding to several values of $\Delta_{1}$. The storage considered here is the live storage. The storage-discharge curves are found to steepen and the maximum live storage under equilibrium condition $(Q=1.0)$ is found to decrease with the increase in the magnitude of $\Delta_{1}$. But for higher magnitudes of $\Delta_{1}$, the maximum live storage is found to be the same and equal to the critical depth under equilibrium condition. The storage-discharge curves are found to follow separate lines in rising and in recession. But while calculating the slope of the above curves average straight line curve is considered.

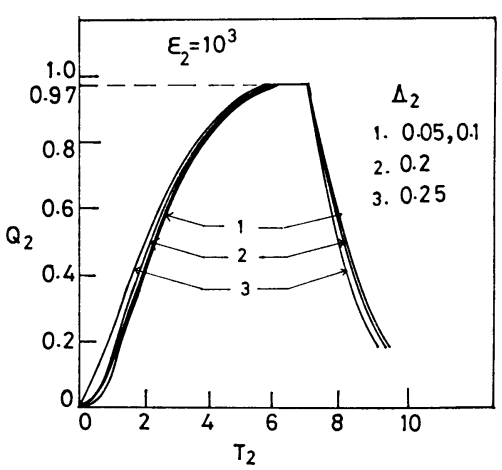

Fig. 8 Channel discharge hydrograph with weir at the outlet for the initial condition $H_{2}=\Delta_{2}$.

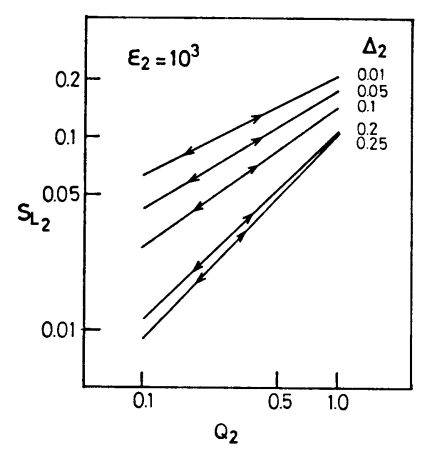

Fig. 9 Logarithmic storage-discharge relation with weir at the channel outlet.

Figures 8 and 9 show the discharge hydrograph and the storage-discharge relation for a channel of $\epsilon_{2}=10^{3}$ with respect to several magnitudes of $\Delta_{2}$. The initial condition of water depth upto the weir crest is considered here. The only inflow to the channel is the lateral outflow from the overland with free fall condition at the outlet.

In Fig. 9 only live storage has been considered. The rising and recession limbs of each storagedischarge curve are found very close and are represented by a single straight line curve. From Figs. 7 and 9 the slope of the storage-discharge curves for both overland and channel are found to vary with $\Delta_{j}$. Figure 10 represents the relation between $\Delta_{j}$ and slope $\alpha$ of the storage discharge curves for the overland and channel systems. Figure 11 shows the semilogarithmic relation between the maximum live storage $S_{L_{\max }}$ and $\Delta j$ with respect to several magnitudes of $\epsilon_{j}$ for both overland and channel systems. It is found that the maximum live storage $S_{L_{\max }}$ for a specified value of $\epsilon$ decreases with the increas- 
ing value of $\Delta_{j}$, but eventually becomes constant and equal to the critical depth for higher magnitudes of $\Delta_{j}$, greater than and equal to $\Delta_{c_{j}}$.

Based on the results stated above, the following non-dimensional general storage discharge equation may be derived for both overland and channel basins.

$$
S_{j}=S_{\max } \cdot Q_{j}^{\alpha}
$$

or

$$
S_{j}=\frac{C_{j}}{\epsilon_{j}^{m}} Q_{j}^{\alpha},
$$

where $S_{j}$ : non-dimensional storage, $Q_{j}$ : nondimensional discharge, $C_{j}$ : storage-discharge coefficient, $\alpha$ : slope of $S-Q$ curve, $m$ : slope of $S_{\max }-\epsilon$ curve, and $S_{\max }=C_{j} / \epsilon_{j}^{m}$ and is the equilibrium storage. Although in this case I only the $S_{L_{\max }}$ is defined and subjected to Eq. (19), but as the equation (19) will be also applied for the next Case II, it is written in terms of the general maximum storage $S_{\max }$.

The above equation with the values $C_{j}=1.0$, $\alpha=0.5$ and $m=0.233$ coincides with the storagedischarge relationship for the free fall outlet condition as stated in the previous paper. But for the case discussed here, we have the other $\alpha$ value depending on the weir height $\Delta$ and the parameter $\epsilon$, as found in Fig. 10. Moreover, Figure 11 shows that the maximum live storage $S_{L_{\max }}$ also depends on the weir height $\Delta$ and $\epsilon$. In the other words, the fundamental form of the storagedischarge relationships remains the same type, but the existence of the weir at the outlet affects the variations of the values of $\alpha$ and $S_{\boldsymbol{L}_{\mathrm{max}}}$ through the values of $\Delta$ and $\epsilon$.

To evaluate the effect of the weir, we will

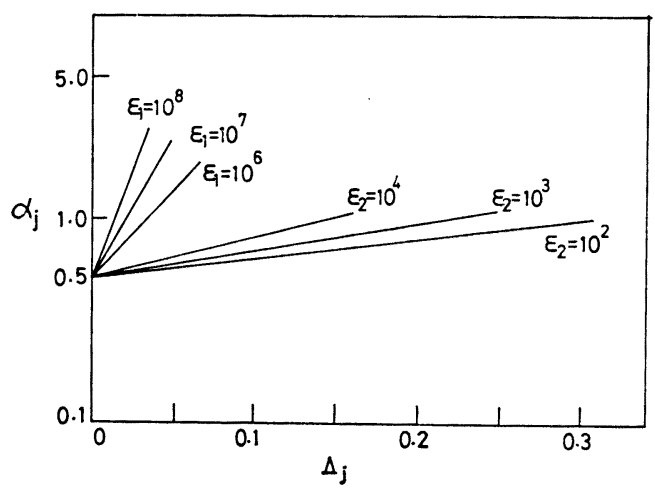

Fig. 10 Semilogarithmic relation between the weir height and the slope of $S-Q$ curve corresponding to several $\epsilon$ for both overland and channel. decompose the values of $\alpha$ and $m$ as follows.

$$
\begin{aligned}
& \alpha=\alpha_{f} \cdot \alpha_{w} \\
& m=m_{f}+m_{w}
\end{aligned}
$$

and

$$
S_{\max }=S_{L_{\max }}=1 /\left(\epsilon^{m_{f}+m_{u}}\right),
$$

where $\alpha_{f}$ : slope of $S-Q$ curve under free fall condition, $\alpha_{w}$ : effect on the slope of $S-Q$ curve due to weir at the outlet, $m_{f}$ : slope of $S_{\max }-\epsilon$ curve under free fall condition and $m_{w}$ : effect on the slope of $S_{\mathrm{max}}-\epsilon$ curve with weir at the outlet. According to Eqs. (19) and (20) $S_{\max }=C_{j} / \epsilon_{j}^{m}$, but if we evaluate the effect of the weir on $m$ as shown in Eq. (22), then $C_{j}$ may be written as $1 / \epsilon^{m_{i v}}$. Therefore, it may also be considered that the effect of weir on the value $C_{j}$ in Eq. (20) is considered through the value $m_{w}$. In the previous study for both overland and channel with free fall conditions at the outlet it is recommended that $C_{j}=1.0, \alpha_{f}=0.5$ and $m_{f}=0.233$. But with weir at the outlet both $\alpha_{w}$ and $m_{w}$ depend on the magnitudes of $\Delta_{j}$ and $\epsilon_{j}$.

For the value of $\alpha_{w}$ the following relation is obtained with the aid of Fig. 10.

$$
\alpha_{w}=\left(\epsilon_{j} / d_{j}\right)^{a_{j} s_{j}}
$$

where $a_{j}$ and $d_{j}$ are constants. As to the value of $m_{w}$, Fig. 11 leads to the following expression.

$$
m_{w}=\left\{\begin{array}{cl}
\frac{\Delta_{j}}{10 \Delta_{c j}} & \text { for } 0 \leq \Delta_{j}<\Delta_{c j} \\
\frac{1}{10} & \text { for } \quad \Delta_{j} \geq \Delta_{c j}
\end{array},\right.
$$

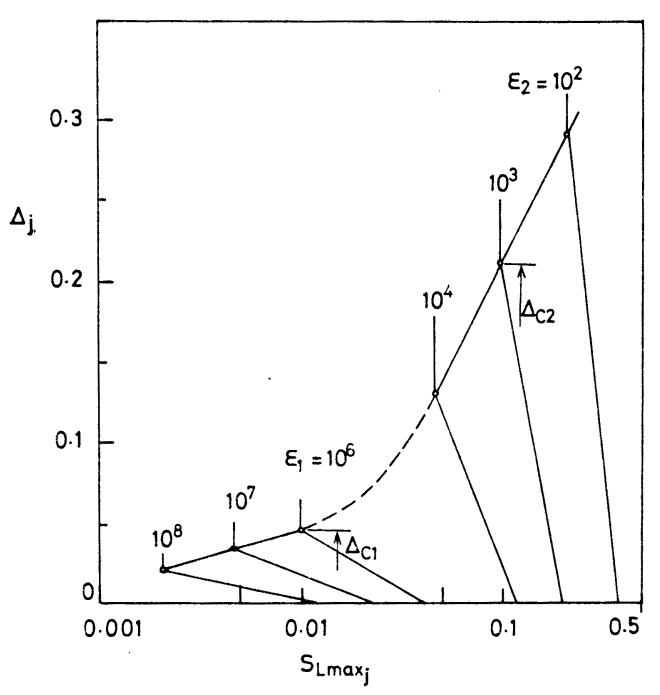

Fig. 11 Semilogarithmic relation between $\Delta_{j}$ and $S_{L_{\max }}$ corresponding to several $\epsilon_{j}$ for both overland and channel. 
where $\Delta_{c j}$ : weir height when $S_{L_{\max }}$ is equal to the critical depth under equilibrium condition. Moreover, $\Delta_{c_{j}}$ in Fig. 11 is assumed to be in linear relation with $S_{L_{\text {max }}}$ for the ranges $\epsilon_{1}=$ $10^{6} \sim 10^{3}$ (overland) and $\epsilon_{2}=10^{2} \sim 10^{4}$ (channel). Then from the relationships between $\Delta_{c_{j}}-\epsilon_{j}$ the following expression is obtained

$$
\Delta_{c j}=K_{j}-p_{j} \log \epsilon_{j},
$$

in which $K_{j}$ and $p_{j}$ are the constant coefficients. In this manner, the storage-discharge relationship is clearly defined, if the several coefficients in Eqs. (24) and (26) are given.

By solving for the coefficients in Eqs. (24) and (26) the following values are obtained and are recommended for practical use.

For the overland

$$
\begin{aligned}
& a_{1}=6, \quad d_{1}=10^{4.5}, \quad K_{1}=0.125, \\
& p_{1}=0.013,
\end{aligned}
$$

For the channel

$$
\begin{aligned}
& a_{2}=0.5, \quad d_{2}=1.0, \quad K_{2}=0.45, \\
& p_{2}=0.08 .
\end{aligned}
$$

\section{(2) Case II: Constant water depth at the out- let}

Figures 12 and 13 show the water profiles and discharge hydrographs for an overland of $\epsilon_{1}=10^{7}$ corresponding to several magnitudes of constant

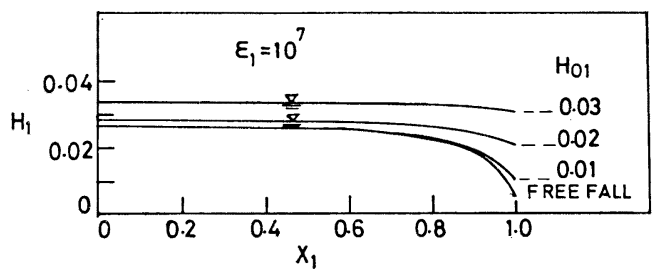

Fig. 12 Equilibrium water profile of the overland for several $H_{01}$.

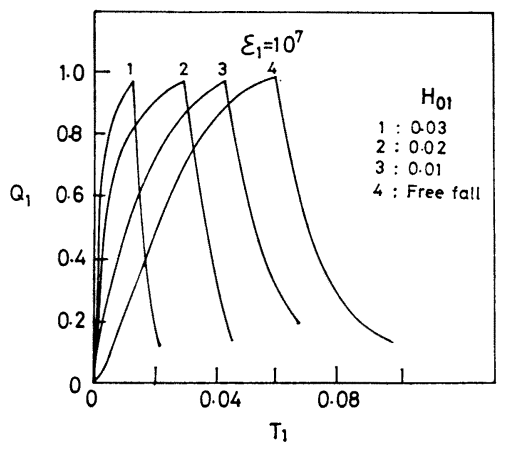

Fig. 13 Overland discharge hydrograph for several $H_{o 1}$. water depth $H_{01}$ at the outlet. The water profile tends to become horizontal with increasing magnitude of $H_{o 1}$. As for the discharge hydrograph, the time to peak is found to decrease with the increase in the value of $H_{01}$. Figure 14 shows the storage-discharge relation for the same overland basin with respect to several magnitudes of $H_{01}$. The slope of the storage-discharge curves decreases with the increase of $H_{01}$ and eventually becomes horizontal for higher values of $H_{o 1}$.

Figure 15 shows the discharge hydrograph for a channel of $\epsilon_{2}=10^{3}$ with constant depth of water $H_{o 2}$ at the channel outlet. The lateral inflow to the channel is the outflow from the overland gathering the rainfall on the overland basin and gradually discharging at the outlet under free fall condition. It is seen that there is negligible variation in the discharge hydrographs for differrent magnitudes of $H_{o 2}$. Figure 16 represents the channel storage-discharge relation for the identical conditions stated above. For both the cases of overland and channel the minimum $H_{o j}$

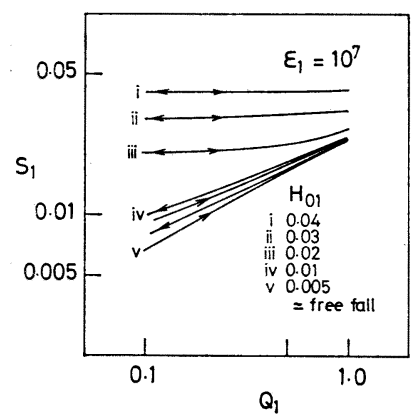

Fig. 14 Logarithmic storage-discharge relation for several $H_{o 1}$-overland.

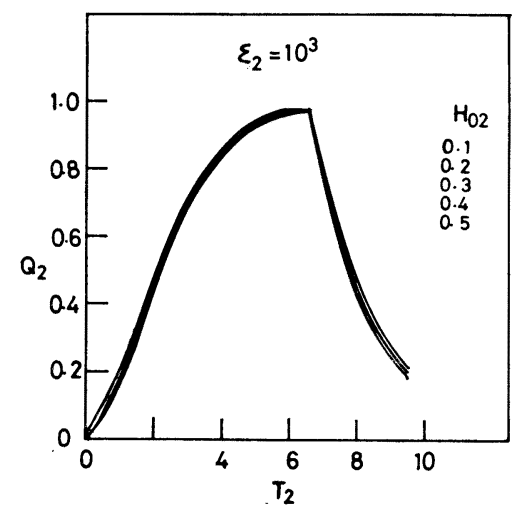

Fig. 15 Channel discharge hydrograph for several $H_{o 2}$. 
considered is the critical depth $H_{c}$.

Each storage-discharge curve in Figs. 14 and 16 is averaged by a straight line curve resulting

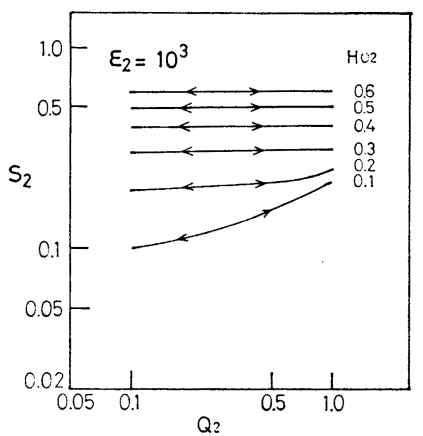

Fig. 16 Logarithmic storage-discharge relation for several $\mathrm{H}_{o 2}$-Channel.

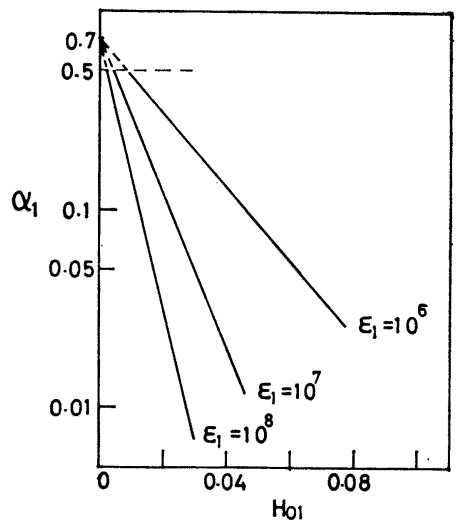

Fig. 17 Semilogarithmic $\alpha-H_{o 1}$ relation for overland.

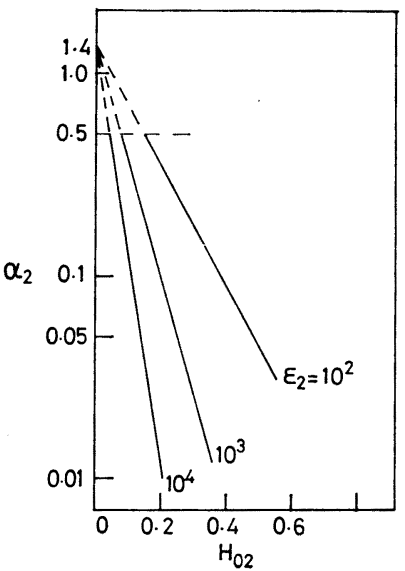

Fig. 18 Semi-logarithmic $\alpha-H_{o 2}$ relation for the channel. the equation (19). Figures 17 and 18 show the semi-logarithmic relations of $\alpha-H_{o 1}$ and $\alpha-H_{o 2}$, respectively for the overland and channel. The curves are found to follow different straight lines for several magnitudes of $\epsilon$.

Figure 19 shows the logarithmic relation between the storage $S_{\max }$ under equilibrium condition and $H_{o j}$ for both overland and channel. All the curves are found to lie between the limits of two straight line curves. The upper straight line curve is represented by $S_{\max }=1 / \epsilon_{j}^{0.233}$ corresponding to the free fall condition when $H_{o j}=H_{c}=1 / \epsilon_{j}^{0.233}$. The lower straight line curve is represented by $S_{\mathrm{max}}=H_{o j}$ corresponding to very large values of $H_{o j}$ when $\alpha \simeq 0$.

Now, with constant depth of water at the outlet, we will consider that the values of $\alpha$ and $S_{\max }$ in Eq. (19) consist of the effect of $H_{o j}$ and a part corresponding to a certain standard state. With respect to the maximum storage $S_{\max }$ with the help of Fig. 19, we introduce here the following expression.

$$
S_{\max }=H_{o j}+D H_{j},
$$

in which $\mathrm{DH}_{j}$ indicates the difference between the ordinate of $S_{\max }-H_{o}$ curve and $S_{\max }=H_{o}$ curve. Then, the relationships between $H_{o j}$ and $H D_{j}$ for the overland and channel are drawn in the semilogarithmic paper as shown in Figs. 20 and 21. These figures deduce the mathematical expression between them, and

$$
D H_{j}=\left(\log \frac{H_{o j}}{H_{o o j}}\right) / m_{H j},
$$

where $H_{o c j}$ indicates the minimum value of $H_{o j}$ for which $D H_{j}=0$ as indicated in Fig. 19. $m_{H j}$

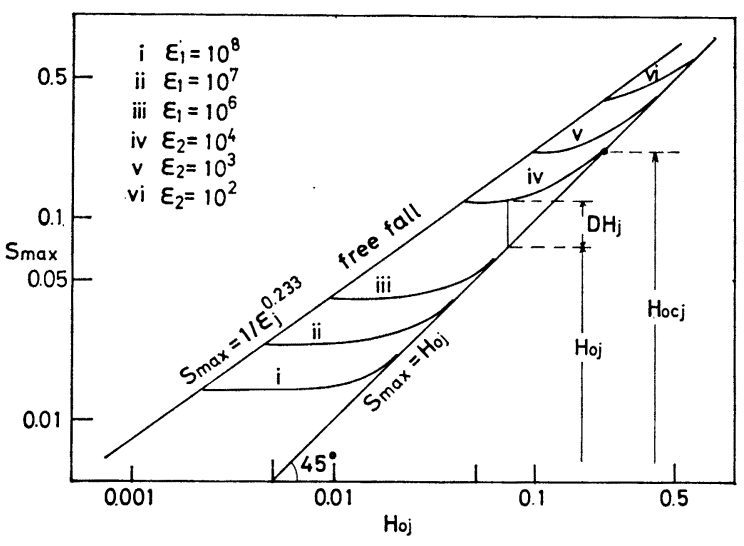

Fig. 19 Logarithmic $S_{\max }-H_{o j}$ relation for both overland and channel. 


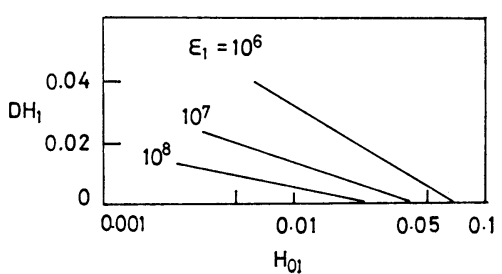

Fig. 20 Semilogarithmic $H_{o 1}-D H_{1}$ relation for overland.

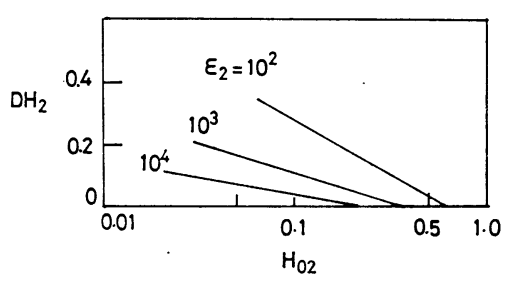

Fig. 21 Semilogarithmic $H_{o 2}-D H_{2}$ relation for the channel.

is the slope of $D H_{j}-H_{o j}$ curve in Figs. 20 and 21. In the above equation when $H_{o j}=H_{o c j}$, $D H_{j}=0$ and as such for the cases $H_{o j} \geq H_{o c j}$, $S_{\mathrm{max}}=H_{o j}$.

Both $H_{o c j}$ and $m_{H j}$ depend on the parameter $\epsilon_{j}$ and are represented by discussing these relationships as follows.

$$
H_{o c j}=\frac{1.78}{\epsilon^{0.233}}=1.78 \cdot S_{f_{\max }}
$$

and

$$
m_{H j}=\log \left(\frac{d_{o j}}{\epsilon_{j}}\right)^{a_{o j}},
$$

where $S_{f_{\mathrm{max}}}=1 / \epsilon_{j}^{0.233}$ and is the equilibrium storage under free fall condition, and $d_{o j}$ and $a_{o j}$ are the coefficients.

While as to the value of $\alpha$, the same form as Eq. (21) is considered.

$$
\alpha=\alpha_{f} \cdot \alpha_{H},
$$

in which $\alpha_{H}$ is the effect on the slope of $S-Q$ curve due to the constant depth of water at the outlet. From Figs. 14, 16, 17 and 18 and with the aid of Eq. (30) following relation with a new coefficient $C_{o j}$ is obtained for $\alpha_{H}$ in Eq. (31).

$$
\alpha_{H}=C_{o j}(10)^{m_{H j} \boldsymbol{H}_{o j}} .
$$

In Eq. (31) the maximum value of $\alpha=\alpha_{f}=0.5$ corresponding to the free fall condition. In that case, the maximum value of $\alpha_{H}$ in Eq. (32) should be 1.0. But in Figs. 17 and 18 the straight line curves are extended beyond $\alpha=0.5$ to have intercept with the $\alpha$-axis. This may give the value of $\alpha_{H}$ in Eq. (32, more than 1.0, but for the reason stated above if $\alpha_{H}>1.0$, put $\alpha_{H}=1.0$. Thus, if the values of the coefficients in Eqs. (30) and (32) are known, the storage-discharge relationship may be established for the basin. By solving for the coefficients in Eqs. (30) and (32) the following values are obtained and are recommended for practical use.

For the overland

$$
d_{o 1}=10^{5}, \quad a_{o 1}=24, \quad C_{o 1}=1.4,
$$

For the channel

$$
d_{o 2}=10, \quad a_{o 2}=3, \quad C_{o 2}=2.8 \text {. }
$$

\section{(3) Case III: Intermediate sections with the free fall at the outlet}

On the Cases I, II and also in the previous paper, we have discussed the storage-discharge relationships. But these are concerned with the discharges at the outlet. Here we will once examine whether the relationships are restricted to the discharge only at the outlet and how the storage-discharge relationship at any intermediate sections along the flow is. As the standard state, the case with free fall condition at the outlet is here considered.

Figure 22 shows the storage-discharge relation at different sections along the overland flow plane. The storage obviously increases with the length of flow plane. All the curves in Fig. 22 are parallel to one another and as such they have the same slope. Similar results are obtained at the different sections along the channel flow length. The storage-discharge relation in Fig. 22 may be represented by Eqs. (19) or (20). In Eq. (20) $\alpha=$ 0.5 and $m=0.233$ are found constants, but the coefficient $C_{j}$ is found to vary along the flow length. Figure 23 shows the relationship between $C_{j}$ and the flow length $X_{j}$ for any magnitude of $\epsilon_{j}$. In this logarithmic plot it is found that $C_{j}$ increases along a straight line upto $80 \%$ of the

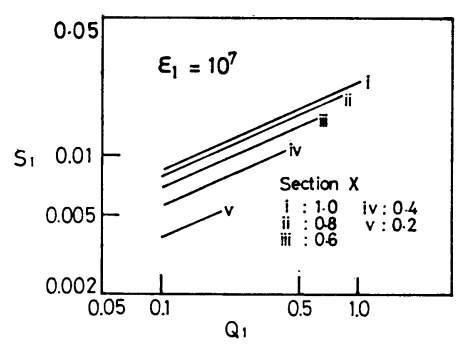

Fig. 22 Logarithmic storage-discharge relation at the intermediate sections for the overland. 


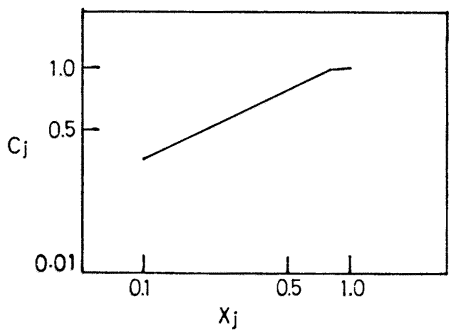

Fig. 23 Logarithmic relation between $C_{j}$ and $X_{j}$ for both overland and channel.

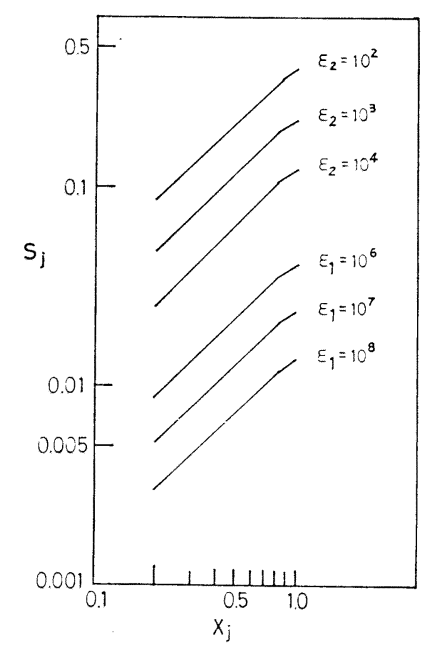

Fig. 24 Logarithmic $S_{\max }-X_{j}$ relation for both overland and channel.

flow length and attains $97 \%$ of its maximum value. After that the curve is found to flatten slightly. Figure 24 shows the relation between $S_{\max }-X_{j}$ for both overland and channel basins corresponding to several magnitudes of $\epsilon_{j}$. Table 1 shows the variation of $C_{j}$ along the flow length of both overland and channel for any magnitude of $\epsilon j$.

\section{DISCUSSION}

\section{(1) Discharge hydrographs}

Case I: Figures 5 and 6 show the overland discharge hydrographs for two extreme cases of initial depths, i.e.

$$
\text { and } \begin{aligned}
H_{j} \mid T_{j=0} & =0 \\
H_{j} \mid T_{j}=0 & =\Delta .
\end{aligned}
$$

For the same $\Delta$, the discharge hydrographs for both the initial conditions are practically the
Table 1 Values of coefficient $m$ and $C$ at intermediate sections for both overland and channel

\begin{tabular}{c|c|c}
\hline$x$ sections & Exponent $m$ & Coefficient $C$ \\
\hline 0.1 & & 0.36 \\
0.2 & & 0.50 \\
0.3 & & 0.61 \\
0.4 & & 0.70 \\
0.5 & & 0.78 \\
0.6 & & 0.85 \\
0.7 & & 0.91 \\
0.8 & & 0.96 \\
0.9 & & 0.98 \\
1.0 & & 1.00 \\
\hline
\end{tabular}

same except for the time lag. The time lag in Fig. 5 for a dry basin is required to fill up the basin upto the crest of the weir before the actual flow starts.

Case II: In case of the overland discharge hydrograph in Fig. 13 the time to peak is found to decrease with the increasing magnitude of the constant depth of water $H_{o 1}$. This may be due to the fact that the $x$-sectional area of flow at the outlet increases with the increase of $H_{01}$ and quickens the flow. But in Fig. 15 the channel discharge hydrographs are found to vary very little with the variation of $H_{02}$. This may be because of the fact that the channel outflow is controlled by the overland outflow which is the only inflow to the channel basin. The overland outflow, which increases with time until equilibrium condition is reached, remains the same for the same overland basin for all magnitudes of constant depth of water $H_{02}$ at the channel outlet.

\section{(2) Storage-discharge relation}

For both overland and channel with different initial and boundary conditions it is found that the storage-discharge relation may be expressed by the following non-dimensional general equation

$$
S=S_{\max } \cdot Q^{\alpha}
$$

For free fall condition at the overland and channel outlets, the magnitudes of $S_{\mathrm{max}}$ and $\alpha$ remain constant for all basins. But for the cases other than free fall at the outlet the magnitudes of the above coefficient and exponent are found to vary depending on the magnitudes of $\Delta_{j}$ and $H_{0 j}$. This is because of the fact that the slope of the storage-discharge curves and the equilibrium storage $S_{\mathrm{max}}$ are found to vary with the variation of $\Delta_{j}$ or $H_{o j}$. Live storage has been considered 
for the case of weir at the basin outlets and in Figs. 7 and $11 S_{L_{\max }}$ is found to decrease with the increase in the magnitude of $\Delta_{j}$, but becomes constant for higher magnitudes of $\Delta j$. This is due to the fact that the water profile gradually flattens and when the water profile becomes horizontal the storage $S_{L_{m a x}}$ is equal to the equilibrium critical depth which is constant for a particular basin.

For the case of $H_{o j}$ at the outlet of the basin, $S_{\max }$ in Fig. 19 is found to increase infinitely with the increasing $H_{o j}$. This is due to the fact that in this case total storage is considered and $H_{o j}$ controls the flow condition. For higher magnitudes of $H_{o j}$, the water profile becomes horizontal and $S_{\mathrm{max}}=H_{o j}$.

\section{(3) The equations and the coefficients}

The equations and the values of the coefficients suggested in this paper for the cases of $\Delta_{j}$ and $H_{o j}$ at the outlet are not as simple as those for the free fall condition at the outlet. But inspite of that these will greatly simplify the solution and understanding of the complicated flow behaviour in the horizontal basin. The values of the coefficients in Eqs. (24) and (26) for $\Delta_{j}$ and in Eqs. (30) and (32) for $H_{o j}$ are obtained from scores of runs and when used show a maximum deviation of around $5 \%$ from the numerical solution for all the values of $\epsilon_{j}, \Delta_{j}$ and $H_{0 j}$ concerned here. This deviation may result due to the fact that all the storage-discharge curves and some of the other curves are averaged as straight lines. The deviation is small and may be neglected.

\section{(4) Hydrograph estimation}

From the principle of continuity the following general basin equation may be written

$$
d s=(R-Q) d t \text {. }
$$

Now, the above Eq. (34) along with the storagedischarge relations for $\Delta_{j}$ or $H_{o j}$ may be solved numerically to obtain the required discharge hydrograph. The non-dimensional discharge hydrographs thus obtained may be converted to the dimensional quantities by multiplying the non-dimensional terms by their normalizing operators.

For hydrograph estimation the variable rainfall in time must be considered. On this point we have already discussed in the reference ${ }^{3)}$ about the two types of variable rainfall e.g. cosine type and triangular type. The results have shown us that the difference in non-dimensional discharge hydrograph between the lumped system and the distributed system has been found as inappreciable, even if we estimate the value of $\epsilon_{j}$ using mean rainfall intensity and apply rainfall input as time-variant.

\section{(5) Practicability of boundary conditions}

Some of the boundary conditions considered in this paper are not usually found in Japan, because of the difference in the drainage requirements and consequent drainage planning. For example, in Bangladesh, with extensive flat lowlying lands, submerged embankments may be constructed along the banks of rivers. The submerged embankments are constructed with the crest level below the normal flood level. But it should be sufficiently high so that the flood water from the river cannot enter the basin before the harvesting of the crops. The rain water is allowed to accumulate inside the basin depending on the crop condition. The accumulated water inside the basin helps in the rural navigation of country boats, rotting of jute plants and killing of the weeds that grow in the agricultural field. When water from the country side flows to the river side over the submerged embankment with river water at lower elevation, the embankment may be considered as a broad-crested weir at the outlet of the overland basin.

The case of constant depth of standing water at the outlet may occur when the runoff from a low-lying overland or channel meets a vast body of wide river or lake with little fluctuation in the water level within short period.

\section{CONCLUSIONS}

The following conclusions may be drawn as a result of this study.

(1) Approrpiate mathematical models with different initial and boundary conditions may be solved by the numerical method and water profile, discharge hydrograph, storage-discharge relation etc. may be obtained for both overland and channel flows.

(2) For both overland and channel with flat beds and with different initial and boundary conditions the non-dimensional storage-discharge relation is expressed by the following general equation

$$
S=S_{\max } \cdot Q^{\alpha}
$$

For free fall condition at the outlet $S_{\mathrm{max}}=1 / \epsilon_{j}^{0.233}$ and $\alpha=0.5$. But for the conditions of weir or constant depth of water at the outlet, the magnitudes of $S_{\max }$ and $\alpha$ are found to vary with the 
weir height or constant depth of water. However, proper expressions could be deduced covering these variations.

(3) For the overland and channel basins with different initial and boundary conditions it is possible to construct discharge hydrographs directly from the rainfall data and basin characteristics.

The solutions, here, are obtained for some basic elemental units for certain boundary conditions. The flow condition in the actual field may be more varied and extensive. But the results obtained here as functional relationships for the basic hydraulic conditions may give us a guideline to understand the flow behaviour and to analyze the runoff phenomena in actual flat lands.

\section{REFERENCES}

1) Chow, V. T.: Open Channel Hydraulics, McGraw Hill Book Company, New York, 1959.

2) Itoh, T.: Application and Fundamentals of Numerical Analysis, Atene Publ. Co., Tokyo, 1971 (in Japanese).

3) Khan, S. I., F. Takagi and S. Adachi: On the Characteristics of runoff in flat land, Proc. JSCE Vol. 289, Sept. 1979, pp. $145 \sim 156$.
4) Liggett, J. A. and Woolhiser, D. A.: Difference solutions of shallow water equations, Journ. of the Engineering Mechanics Division, ASCE, Vol. 93, No. EM 2, Proc. Paper 5189, pp. 39 71, April 1967.

5) Morgali, J. R. and R. K. Linsley: Computer Analysis of Overland Flow, Journ. of ASCE, Vol. 91, No. HY 3, Proc. Paper 4325, pp. 81 100, May 1965.

6) Morgali, J. R.: Laminar and Turbulent overland flow hydrographs, Jour. ASCE, Vol. 96, No. HY 2, Proc. Paper 7069, pp. 441 460, Feb. 1970.

7) Suzuki, M. and I. Nishihata: Study on runoff analysis in low-lying land, Proc. JSCE, Vol. 163 pp. 29 37, March 1969 (in Japanese).

8) Toyokuni, E., M. Kadoya and G. Ohhashi: Runoff Characteristics of Yamashina river basin, Annuals of Disaster-Prevention Research Institute of Kyoto University, No. 8, pp. 297 306, March 1965 (in Japanese).

9) Toyokuni, E. and M. Kadoya: Runoff analysis in paddy field district, Annuals of Disaster Prevention Research Institute of Kyoto University, No. 9, pp. 631 636, March 1966 (in Japanese).

(Received June 14, 1979) 